\title{
MicroRNA-448 modulates the progression of neuropathic pain by targeting sirtuin 1
}

\author{
YUNCHAO CHU $^{1 *}$, WEIPENG GE ${ }^{1 *}$ and XIN WANG ${ }^{2}$ \\ Departments of ${ }^{1}$ Pain Treatment and ${ }^{2}$ Anesthesiology, Shengli Oilfield Central Hospital, \\ Dongying, Shandong 257034, P.R. China
}

Received February 21, 2019; Accepted October 11, 2019

DOI: $10.3892 /$ etm.2019.8165

\begin{abstract}
MicroRNAs (miRNAs) play crucial roles in the pathogenesis of neuropathic pain. The present study investigated the effects of miR-448 on the progression of neuropathic pain in a rat model of chronic constriction injury (CCI) of the sciatic nerve. Reverse-transcription quantitative polymerase chain reaction was conducted to detect the gene expression. The paw withdrawal threshold (PWT) and paw withdrawal latency (PWL) were used to assess the pain threshold. The protein expression levels of interleukin (IL)-6, IL-1 $\beta$ and tumor necrosis factor- $\alpha$ (TNF- $\alpha$ ) were detected by ELISA. The target of miR-448 was predicted by TargetScan software. The Student's t-test or one-way ANOVA were used to identify statistical differences among groups. miR-448 was persistently upregulated in CCI rats, and both mechanical allodynia and thermal hyperalgesia in CCI rats were decreased following miR-448 downregulation. The expression levels of IL-1 $\beta$, IL- 6 and TNF- $\alpha$ were significantly increased in $\mathrm{CCI}$ rats compared with controls, and these effects were reversed following treatment with a miR-448 inhibitor. A luciferase reporter assay revealed that sirtuin 1 (SIRT1) was a target gene of miR-448. SIRT1 was found to abrogate the effect of miR-448 on neuropathic pain development. Collectively, the results of the present study revealed that miR-448 promoted neuropathic pain in CCI rats by regulating neuroinflammation via SIRT1. Therefore, SIRT1 may be considered as a novel biomarker for neuropathic pain.
\end{abstract}

\section{Introduction}

Neuropathic pain is caused by nerve dysfunction or lesions (1), and is caused by the response of the central and peripheral

Correspondence to: Dr Xin Wang, Department of Anesthesiology, Shengli Oilfield Central Hospital, 31 Jinan Road, Dongying, Shandong 257034, P.R. China

E-mail: baoyu45213133@163.com

*Contributed equally

Key words: microRNA-448, neuropathic pain, neuroinflammation, sirtuin 1 nervous systems to the nerve injury (2). Neuropathic pain is regarded as a serious public health concern worldwide and results in poor quality of life for patients (3). Various factors, such as surgery, trauma, toxicity and congenital disorders, can exacerbate neuropathic pain (4). However, no effective treatments have been established for neuropathic pain. Therefore, it is of great importance to explore the molecular mechanism of this condition.

MicroRNAs (miRNAs) are a class of small and non-coding RNA molecules, which serve as post-transcriptional regulators of gene expression by binding to the 3'-untranslated region (UTR) of target mRNAs (5). Numerous studies have suggested that miRNAs are involved in many biological processes, including cell development, proliferation, differentiation, apoptosis and the stress response (6-8). A number of aberrantly expressed miRNAs have been identified in various types of human cancer $(9,10)$. Moreover, several miRNAs have recently been reported to participate in the development of neuropathic pain. For instance, miR-98 suppresses the progression of neuropathic pain by targeting high mobility group A2 (11). miR-206-3p acts as an inhibitor of chronic constriction injury-induced neuropathic pain by regulating the expression of histone deacetylase 4 (12). miR-28-5p attenuates the development of neuropathic pain by binding to the 3'-UTR of zinc finger E-box-binding homeobox in vivo (13). Notably, Lu et al (14) reported that miR-448 was differentially expressed in rat cortex following anesthesia with sevoflurane, suggesting a role for miR-448 in neurological function. On basis of these results, the present study investigated the role of miR-448 in the progression of neuropathic pain.

\section{Materials and methods}

Animal studies. A total of 62 adult female Sprague-Dawley rats with weights ranging from 180 to $210 \mathrm{~g}$ were purchased from the Shanghai Animal Laboratory Center. All rats were housed in individual cages with a constant temperature of $25^{\circ} \mathrm{C}$ and had free access to food and water. The health and behavior of the rats were monitored every day. A rat model of neuropathic pain was established by chronic constriction injury (CCI) as previously described (15). A total of 30 rats were randomly divided into the sham group and CCI group ( $n=15$ rats per group). Rats were anesthetized by the intraperitoneal injection of $40 \mathrm{mg} / \mathrm{kg}$ sodium pentobarbital. The 
sciatic nerves on both sides were exposed and separated from the surrounding tissues. Sham-operated rats were subjected to sciatic nerve exposure and isolation without ligation. The rats were euthanized on days $0,3,7,14$ and 21 following surgery by the intravenous administration of $140 \mathrm{mg} / \mathrm{kg}$ pentobarbital. Total cardiac arrest, pupil dilation, muscle flaccidity and absence of reflexes were used to confirm euthanasia. As the segmental nerves L4-L6 contribute to the sciatic nerve (16), the dorsal spinal cords of these nerves were collected immediately following euthanasia.

The experimental procedures for care and use of animals were approved by the Medical Ethics Committee of Shengli Oilfield Central Hospital (reference number, 20170016), and were performed strictly under the requirements of the Guide for the Care and Use of Laboratory Animals of the National Institutes of Health. Humane endpoints were followed in accordance with the OECD Guidance Document on the Recognition, Assessment and Use of Clinical Signs as Humane Endpoints for Experimental Animals Used in Safety Evaluation (2000), and measures were taken to minimize pain and discomfort, such as a cut-off time for the pain threshold assessment.

Cell culture. Rat microglial cells (HAPI) were obtained from the American Type Culture Collection. All cells were cultured in DMEM (Lonza Group Ltd.) supplemented with $10 \%$ fetal bovine serum (Invitrogen; Thermo Fisher Scientific, Inc.) and $1 \%$ penicillin/streptomycin. Cells were incubated in a $5 \% \mathrm{CO}_{2}$ incubator at $37^{\circ} \mathrm{C}$. The miR-448 mimic, miR-448 inhibitor and scrambled controls were purchased from Shanghai GenePharma Co., Ltd. The sequences were as follows: miR-448 mimic, 5'-UUGCAUAUGUAGGAUGUCCCA U-3'; miR-448 mimic NC, 5'-UUCUCCGAACGUGUCACG UTT-3'; miR-448 inhibitor, 5'-AUGGGACAUCCUACAUAU GCAA-3'; miR-448 inhibitor NC, 5'-CAGUACUUUUGU

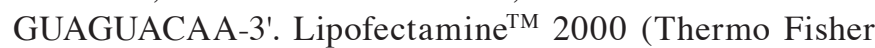
Scientific, Inc.) was used to transfect the miRNAs (100 nM) into HAPI cells according to the manufacturer's protocol. At $48 \mathrm{~h}$ post-transfection, the cells were harvested for the subsequent experiments.

Lentivirus $(L V)$ production and infection. Lentiviruses delivering short hairpin (sh) RNAs against miR-448 [LV-miR-448 knockdown (KD)], sirtuin 1 [SIRT1 (LV-SIRT1 KD)] and their respective scrambled sequences (LV-miR-scrambled) were purchased from Shanghai GenePharma Co., Ltd.

A total of 32 rats were randomly assigned into four groups: Sham group, CCI + LV-miR-scrambled, CCI + LV-miR-448 $\mathrm{KD}$ group and CCI + LV-miR-KD + SIRT1 KD group $(\mathrm{n}=8$ per group). A PE-10 polyethylene catheter was inserted into the rats to allow the delivery of the lentiviral vectors. A volume of $10 \mu \mathrm{l}$ of $0.5 \%$ lidocaine $(0.9-1.1 \mathrm{mg} / \mathrm{kg})$ was injected into rats through an intrathecal catheter. The catheter was confirmed to be successfully inserted when paralysis of the bilateral hind limbs arose immediately after anesthesia. Then intrathecal catheter was also used for lentivirus injection $\left(1 \times 10^{8} \mathrm{PFU} / \mathrm{ml}\right.$, $10 \mu \mathrm{l})$. All rats were euthanized 7 days postoperatively.

RNA extraction and reverse-transcription quantitative polymerase chain reaction $(R T-q P C R)$. Total RNA was isolated from tissues or cells using a miRNeasy mini kit (Qiagen) according to the manufacturer's protocol. RT was performed using TaqMan miRNA reverse-transcription kit (Applied Biosystems; Thermo Fisher Scientific, Inc.) according to the manufacturer's protocol. qPCR was conducted to detect the expression levels of miR-448 and SIRT1 using a SYBR Premix Ex Taq ${ }^{\mathrm{TM}}$ II commercial kit (Takara Biotechnology Co. Ltd.) and the Applied Biosystems 7900 Real-Time PCR System (Applied Biosystems; Thermo Fisher Scientific, Inc.) according to the manufacturer's protocol. The PCR conditions were as follows: $95^{\circ} \mathrm{C}$ for $5 \mathrm{~min}$, followed by 40 cycles of $95^{\circ} \mathrm{C}$ for $30 \mathrm{sec}, 60^{\circ} \mathrm{C}$ for $30 \mathrm{sec}$, and $72^{\circ} \mathrm{C}$ for $40 \mathrm{sec}$ and then a final extension at $72^{\circ} \mathrm{C}$ for $5 \mathrm{~min}$. mRNA levels were quantified using the $2^{-\Delta \Delta \mathrm{Cq}}$ method and normalized to the internal reference gene GAPDH or U6 (17). Primers used in this study are as follows: miR-448, forward, 5'-GCCGAGTTGCATATG TAGGA-3' and reverse, 5'-ATGCATGCCACGGGCATATAC ACT-3'; SIRT1, forward, 5'-ATGAAGCACCAACCGTAT C-3' and reverse, 5'-CTGAATTGACCTTGACTGATG-3'; U6, forward, 5'-CGCTTCGGCAGCACATATACTAAAATTGGA AC-3' and reverse, 5'-GCTTCACGAATTTGCGTGTCATCC TTGC-3'; GAPDH: forward, 5'-AGAAGGCTGGGGCTCATT TG-3' and reverse, 5'-AGGGGCCATCCACAGTCTTC-3'.

Pain threshold assessment. Behavioral testing was conducted between 9:00 a.m. and 4:00 p.m on days $0,3,7,14$ and 21 following surgery. The paw withdrawal threshold (PWT) was used to calculate mechanical allodynia using a calibrated electronic von Frey filament $\left(0.5 \times 0.5 \mathrm{~cm}^{2}\right.$; cat. no. 2393 ; IITC Inc. Life Science) on and the plantar surface of each hind paw. The time taken to elicit the paw withdrawal response was evaluated. Paw withdrawal latency (PWL) was used to evaluate the thermal hyperalgesia response to radiant heat using a thermal paw stimulation system (BME-410C; Institute of Biomedical Engineering, Chinese Academy of Medical Sciences). The animals were exposed to heat for $10 \mathrm{sec}$ and the experiment had a cut-off time of $20 \mathrm{sec}$ regardless of whether the animals reacted or not. The duration between the start of the stimulus and paw withdrawal was recorded.

ELISA. The frozen spinal cord tissues (30 mg) were dissolved in $300 \mu 1$ RIPA lysis buffer (Beyotime Institute of Biotechnology) at $4^{\circ} \mathrm{C}$ for $3 \mathrm{~h}$ and the homogenate was then centrifuged at $12,000 \mathrm{x} \mathrm{g}$ for $10 \mathrm{~min}$ at $4^{\circ} \mathrm{C}$ to remove debris. The protein expression levels of interleukin (IL)-6 (cat. no. R6000B), IL-1 $\beta$ (cat. no. RLB00) and tumor necrosis factor- $\alpha$ (TNF- $\alpha$, cat. no. RTA00) in the tissue homogenate was then dermined using ELISA kits according to the manufacturer's protocols (R\&D Systems China Co., Ltd.).

Luciferase activity assay. The target gene of miR-448 was predicted using TargetScan 7.1 bioinformatics software (www.targetscan.org/vert_71). The wild-type (WT) or mutant (Mut) SIRT1 binding site in miR-448 was amplified and sub-cloned into the pGL3 basic vector (Promega Corporation). Next, $50 \mathrm{ng}$ of pGL3 vectors and $200 \mathrm{ng}$ of miR-448 mimics or miR-448 inhibitor were co-transfected into rat microglial cells using Lipofectamine ${ }^{\mathrm{TM}} 2000$ reagent (Invitrogen; Thermo Fisher Scientific, Inc.). Cells transfected with only Lipofectamine ${ }^{\mathrm{TM}} 2000$ reagent were used as the 
mock transfection group. After $48 \mathrm{~h}$, Luciferase activity was measured using a Dual-Luciferase Reporter Assay system (E1910; Promega Corporation) according to the manufacturer's instructions. Renilla luciferase activity was used as the internal control.

Statistical analysis. The data are presented as mean \pm standard deviation. Differences between two groups were compared using Student's t-test. One-way ANOVA analysis followed by Tukey's post hoc test was used for the comparison of two or more groups. All data analyses were conducted using SPSS software (version 18.0; SPSS Inc.) and GraphPad Prism software (version 5.0; GraphPad Software, Inc.). $\mathrm{P}<0.05$ was considered to indicate a statistically significant difference.

\section{Results}

Expression of miR-448 is significantly increased in a rat model of CCI. To investigate the role of miR-448 in neuropathic pain development, the expression level of miR-448 was first measured in the spinal cords of CCI and sham-operated rats. As shown in Fig. 1, the expression level of miR-448 increased significantly in a time-dependent manner in CCI compared with sham-operated rats $(\mathrm{P}<0.05)$.

miR-448 downregulation inhibits the development of neuropathic pain. As miR-448 was persistently expressed in CCI rats, the downregulation of miR-448 may attenuate the development of neuropathic pain. To test this hypothesis, an miR-448 inhibitor was intrathecally administered to the rats. Compared with that in the sham-operated group, miR-448 was significantly upregulated in CCI rats, and both the PWT and PWL were decreased significantly in rats with CCI (all $\mathrm{P}<0.05$; Fig. 2). Compared with $\mathrm{CCI}+$ scrambled group, miR-448 was significantly downregulated in CCI rats treated with the miR-488 inhibitor $(\mathrm{P}<0.05$; Fig. 2A). Additionally, the PWT and PWL were lower in CCI rats compared with sham-operated group, and these effects were abrogated by miR-448 downregulation ( $\mathrm{P}<0.05$; Fig. 2B-C).

miR-448 downregulation depresses inflammatory cytokines levels in vivo. To further investigate the biological effect of miR-448 on neuropathic pain, the expression levels of inflammatory cytokines were measured in CCI rats when miR-448 was downregulated. The protein levels of IL-1 $\beta$, IL- 6 and TNF- $\alpha$ were significantly increased in rats with CCI compared with those in the sham-operated group, and the levels were significantly decreased following treatment with the miR-448 inhibitor (all $\mathrm{P}<0.05$; Fig. 3).

miR-448 directly targets the 3'UTR of SIRT1. The potential mechanism of miR-448 in regulating neuropathic pain was further explored, and the potential target gene of miR-448 was investigated. The TargetScan analysis suggested that the 3'-UTR of SIRT1 contained a binding region for miR-448 (Fig. 4A). To confirm the transfection efficiency of miR-448 mimic or inhibitor, the relative expression of miR-448 was quantified using RT-qPCR. It was noted that miR-448 mimic transfection significantly increased miR-448 level in HAPI cells compared with the miR scrambled control, whereas

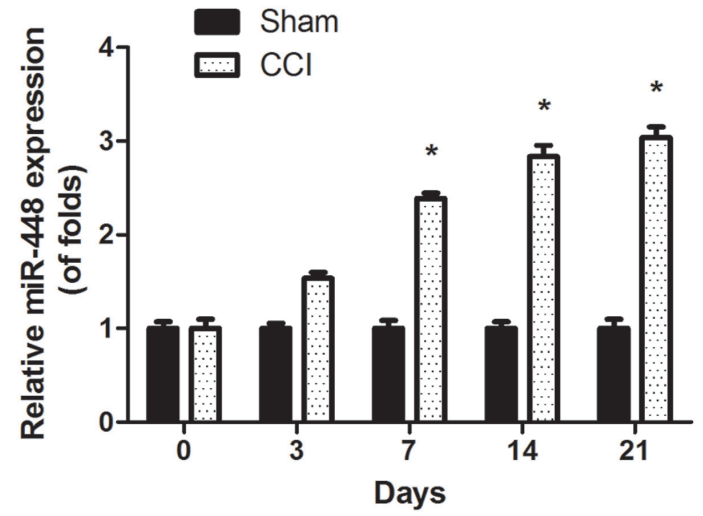

Figure 1. miR-448 expression in the L4-L6 dorsal spinal cord of CCI and sham-operated rats was compared at each time-point. Reverse transcription-quantitative polymerase chain reaction was performed on post-operative days $0,3,7,14$ and 21. Data are presented as the mean \pm SD of at least three experiments. ${ }^{*} \mathrm{P}<0.05$ vs. the sham group at the indicated time-points. miR, microRNA; CCI, chronic constriction injury.

miR-448 inhibitor transfection significantly decreased the miR-448 expression level $(\mathrm{P}<0.05$; Fig. 4B). The luciferase reporter assay suggested that treatment with the miR-448 mimic significantly attenuated the luciferase activity in WT SIRT1 3'UTR transfected cells, compared with treatment with miR-scrambled control, while the inhibition of miR-448 promoted luciferase activity (Fig. 4C). However, the miR-448 mimic did not affect the luciferase activity in Mut SIRT1 3'UTR transfected cells. Additionally, the RT-qPCR analysis results showed that the reduced SIRT1 mRNA expression in rats with CCI was significantly upregulated following miR-448 inhibitor treatment $(\mathrm{P}<0.05$; Fig. 4D).

Effect of miR-448 on neuropathic pain is reversed by SIRT1. To detect whether miR-448 affects the development of neuropathic pain by regulating SIRT1 expression, rescue experiments were performed. SIRT1 mRNA expression levels were detected in rats subjected to CCI co-infected with miR-448 inhibitor and shSIRT1 lentiviruses. The increased SIRT1 mRNA expression level induced by miR-448 inhibitor treatment was significantly decreased following shSIRT1 infection $(\mathrm{P}<0.05$; Fig. 5A). Furthermore, the suppressive effects of the miR-448 inhibitor on mechanical allodynia and thermal hyperalgesia were substantially reversed by shSIRT1 infection (Fig. 5B-C; all $\mathrm{P}<0.05)$. Additionally, the decreased expression of inflammatory cytokines, including IL- $1 \beta$, IL- 6 and TNF- $\alpha$, caused by the miR-448 inhibitor, was also reversed by shSIRT1 (all $\mathrm{P}<0.05$; Fig. 5D-F). The data indicated that miR-448 enhanced neuropathic pain by targeting SIRT1.

\section{Discussion}

Neuropathic pain can be caused by different neurological diseases resulting from injury of the peripheral nerves. Patients with cancer, diabetes and acquired immunodeficiency syndrome are likely to suffer from neuropathic pain (18-20). It has been noted that the expression of IL-1 $\beta$, IL-6 and TNF- $\alpha$ can be induced by nerve surgery (21), indicating that inflammation could play a crucial role in the progression of neuropathic pain (22). 
A

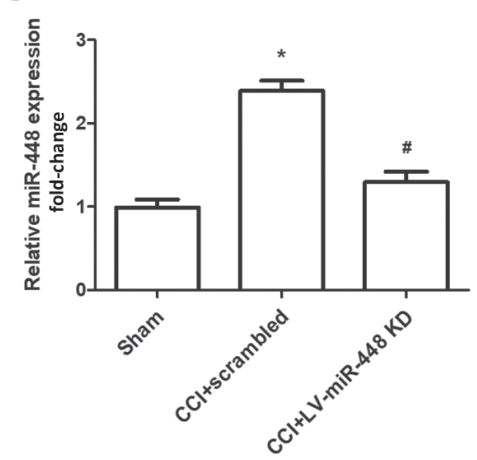

B

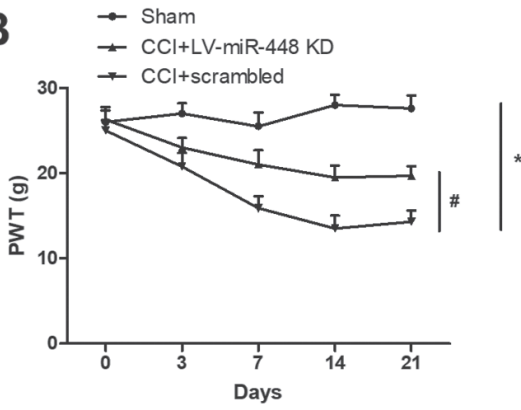

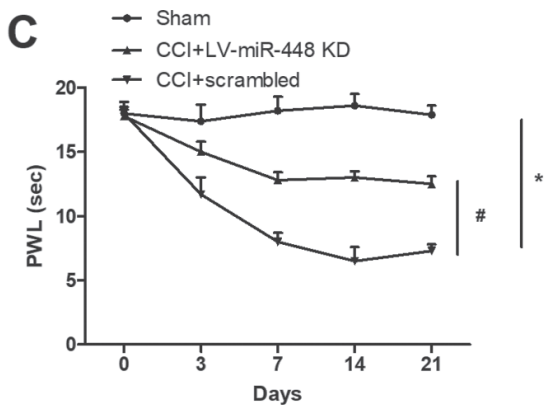

Figure 2. Effects of miR-448 on mechanical allodynia and thermal hyperalgesia in CCI rats. (A) Expression of miR-448 in CCI models infected with miR-scramble or miR-448 inhibitor on post-operative day 7. (B) The effect of downregulated miR-448 expression on mechanical allodynia was assessed by the PWT, indicated in $\mathrm{g}$. (C) The effect of the miR-448 inhibitor on thermal hyperalgesia indicated by PWL, indicated in sec. " $\mathrm{P}<0.05$ vs. the sham group; ${ }^{\#} \mathrm{P}<0.05$ vs. the $\mathrm{CCI}+$ scrambled group. miR, microRNA; CCI, chronic constriction injury; PWT, paw withdrawal force; PWL, paw withdrawal latency.

A

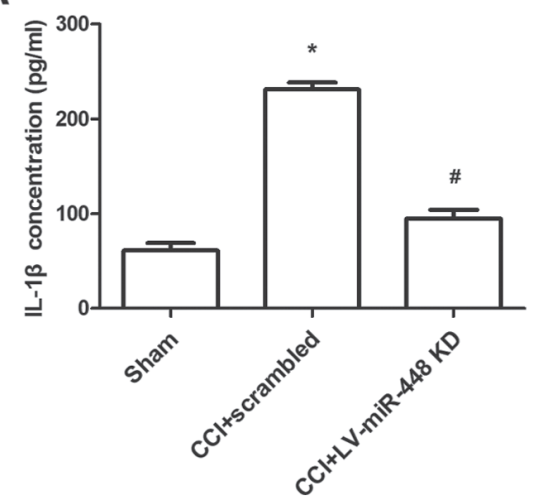

B

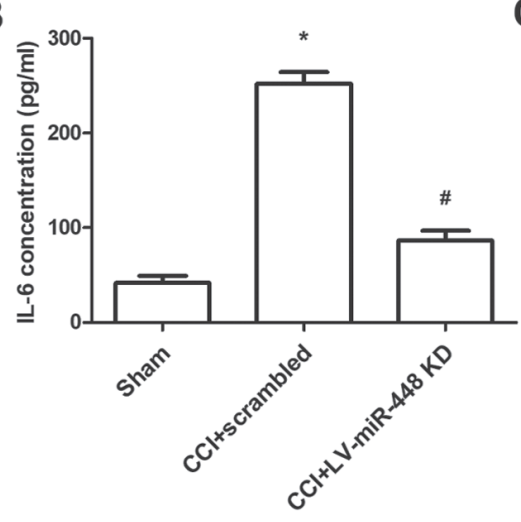

C

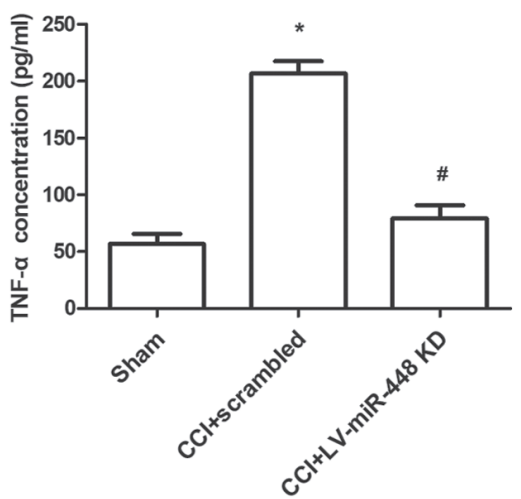

Figure 3. Treatment with the miR-448 inhibitor suppressed neuroinflammation in rats with chronic constriction injury. The protein levels of (A) IL-1 $\beta$, (B) IL-6 and (C) TNF- $\alpha$ in the spinal cords of rats treated with miR-448 inhibitor or scrambled control were measured using ELISA 7 days post-surgery. ${ }^{*} \mathrm{P}<0.05$ vs. the sham group; ${ }^{\prime} \mathrm{P}<0.05$ vs. the $\mathrm{CCI}+$ scrambled group. miR, microRNA; KD, knockdown; IL, interleukin; TNF- $\alpha$, tumor necrosis factor- $\alpha$; LV, lentivirus.

miRNAs are a class of non-coding RNAs 18-24 nucleotides in length. A number of miRNAs have been shown to be aberrantly expressed in various types of diseases, including inflammatory diseases, heart diseases and several types of human cancer $(23,24)$. Increasing evidence has reported that miRNAs may be involved in the development of neuropathic pain development $(11,12)$. Altered expression of miR-448 has been detected in various human diseases. For instance, miR-448 was expressed at low levels in glioma cells, and suppressed cell proliferation and promoted apoptosis (25). Zhang et al (26) suggested that miR-448 was highly expressed in vascular smooth muscle cells (VSMCs) from coronary atherosclerotic plaques, and overexpression of miR-448 promoted VSMC migration. Hong et al (27) indicated that miR-448 is upregulated in gastric cancer tissues compared with adjacent non-tumor samples, and can enhance the glycolytic metabolism of gastric cancer cells by downregulating lysine demethylase 2B (KDM2B). All evidence suggests the various roles of miR-448 in different diseases. In the present study, miR-448 was significantly increased in CCI rat compared with the sham-operated group, and downregulation of miR-448 markedly attenuated the progression of neuropathic pain. A previous study suggested that miR-448 was upregulated in the spinal cord tissues of spinal cord ischemia/reperfusion injury model rats, as well as in cells treated with hypoxia and that downregulation of miR-448 inhibited apoptosis in nerve cells and improved neurological function (28). Additionally, Lu et al (14) reported that the expression level of miR-448 was significantly upregulated in rats treated with sevoflurane compared with controls (14). An et al indicated that lead exposure resulted in the upregulation of miR-448 in rat models (29). The aforementioned studies support the results obtained in the present study.

Previous evidence has suggested that neuroinflammation plays a crucial role in the development of neuropathic pain (30). IL-1 $\beta$, IL-6 and TNF- $\alpha$ are commonly implicated as inflammation-associated cytokines $(31,32)$. In the present study, the ELISA results suggested that the expression levels of IL-1 $1 \beta$, IL- 6 , and TNF- $\alpha$ were decreased in the spinal cords of rats following treatment with the miR-448 inhibitor. This suggested that the downregulation of miR-448 markedly decreased the progression of neuropathic pain through by attenuating neuroinflammation.

miRNAs exert their functions by binding to the target genes at a post-transcriptional level $(33,34)$. Previous evidence has suggested that miR-448 exhibits differing functions in human diseases. For example, Su et al (25) revealed that miR-448 suppresses cell proliferation and promotes apoptosis in glioma 
A

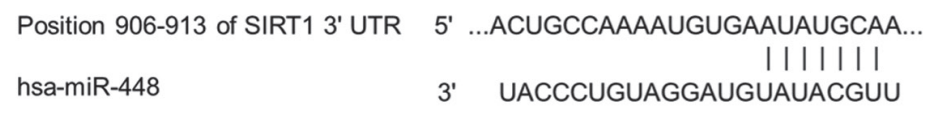

B
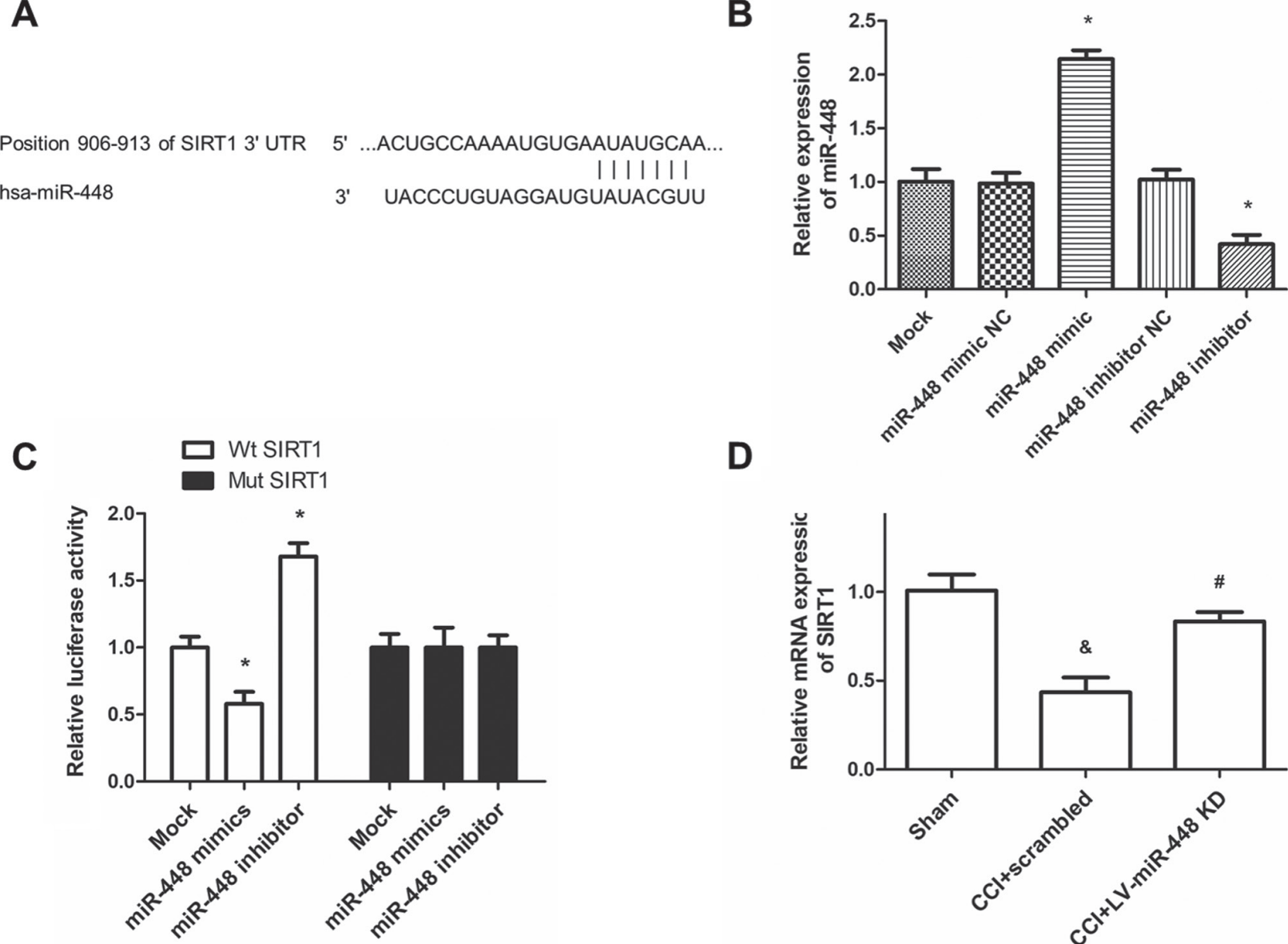

D

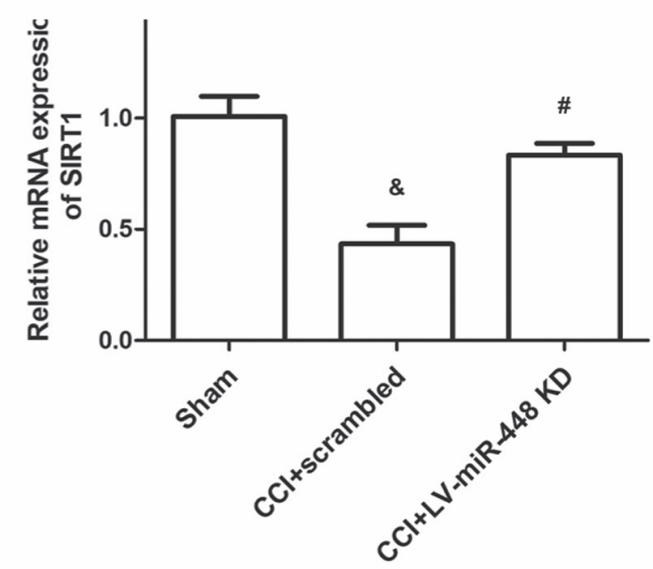

Figure 4. miR-448 directly targeted the SIRT1 3'-UTR. (A) Depiction of the predicted miR-448 binding sites within the SIRT1 3'-UTR. (B) Relative expression of miR-448 in cells transfected with a miR-448 mimic or inhibitor. (C) The luciferase activity of WT or Mut SIRT1 3'-UTR in rat microglial cells transfected with a miR-448 mimic or inhibitor. (D) Effect of the miR-448 inhibitor on the mRNA expression of SIRT1. The reduced SIRT1 mRNA expression in rats with CCI was significantly upregulated following treatment with the miR-448 inhibitor. ${ }^{*} \mathrm{P}<0.05$ vs. the mock group; ${ }^{\circ} \mathrm{P}<0.05$ vs. the sham group; ${ }^{\#} \mathrm{P}<0.05$ vs. the CCI + scrambled group. miR, microRNA; 3'-UTR, 3'-untranslated region; SIRT1, sirtuin 1; WT, wild-type; Mut, mutant; KD, knockdown; CCI, chronic constriction injury.

by downregulating cortactin. Yang et al (35) reported that miR-448 contributes to the development of osteoarthritis by downregulating matrilin-3 expression. Zhang et al (26) suggested that overexpression of miR-448 enhanced cell proliferation and migration in vascular smooth muscle cells (VSMCs) by targeting MEF2C. Previous studies reported that several genes are target genes of miR-448 (29,36); however, only one of those target genes, SIRT1, was investigated in the present study. For example, Hong et al (27) reported that miR-448 can promote glycolytic metabolism in gastric cancer, and KDM2B was revealed to be the target gene that can repress glycolysis. Insulin like growth factor 1 receptor (IGF1R) was reported to be the target gene of miR-448 in colorectal cancer cells and IGF1R gene overexpression reversed the suppressive effect of miR-448 on tumor progression in vitro (36). Additionally, Wang et al (28) suggested that downregulation of miR-448 can relieve SCII by upregulating SIRT1. In the present study, luciferase reporter assay results confirmed that SIRT1 was the target of miR-448. Additionally, rescue experiments were performed to detect whether miR-448 affects the development of neuropathic pain by regulating SIRT1 expression. Overexpression of SIRT1 reversed the effect of miR-448 on neuropathic pain development. Therefore, miR-448 may enhance neuropathic pain by targeting SIRT1. A major study reported that downregulation of miR-448 relieved SCII, inhibited apoptosis of nerve cells and improved neurological function by upregulating SIRT1 (28), which was in accordance with the results obtained in the present study.

The neuroprotective effect of SIRT1 has been widely investigated in previous studies. Fujita and Yamashita (37) suggested that SIRT1 is involved in the protection of neurons from degeneration in models of neurological diseases, including traumatic brain injury and Alzheimer's disease (37). Zhao et al (38) suggested that the expression level of SIRT1 was increased in resveratrol-treated animals, and exerted neuroprotective effects on spinal cord injury. Additionally, Zhang et al (39) reported that SIRT1 alleviates neuropathic pain in rats with type 2 diabetes mellitus. Furthermore, a recent study reported that downregulation of miR-377 could decrease proinflammatory cytokine release through upregulation of SIRT1 (40). SIRT1 has also been reported to regulate the secretion of inflammatory factors induced by high glucose or hypoxia (41), suggesting the crucial role of SIRT1 in host inflammatory responses. The aforementioned studies indicate that SIRT1 exhibits a neuroprotective role by suppressing neuroinflammation, which was consistent to the results obtained in the present study. 
A

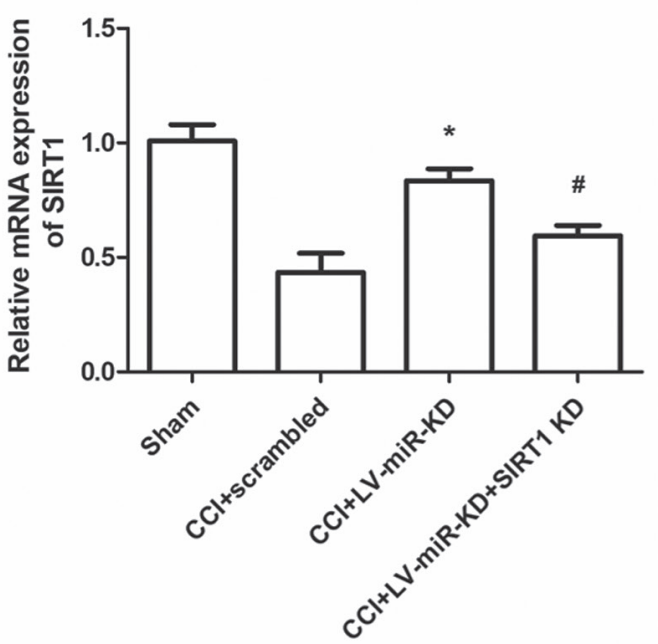

C

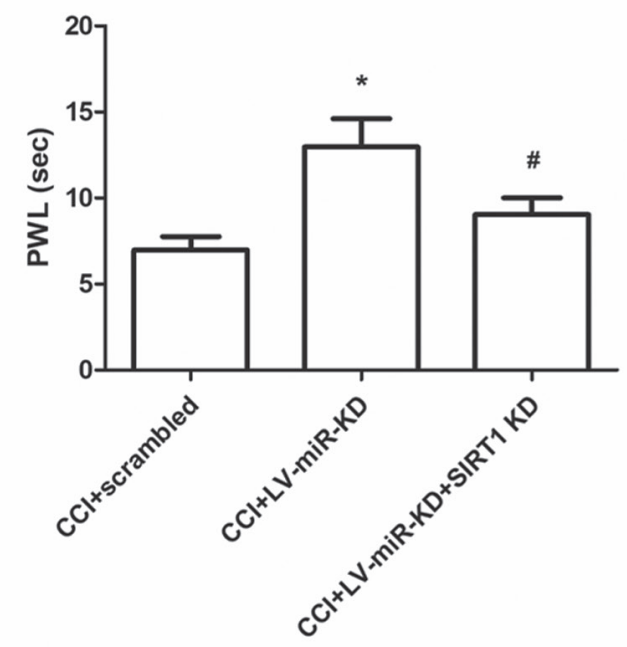

E

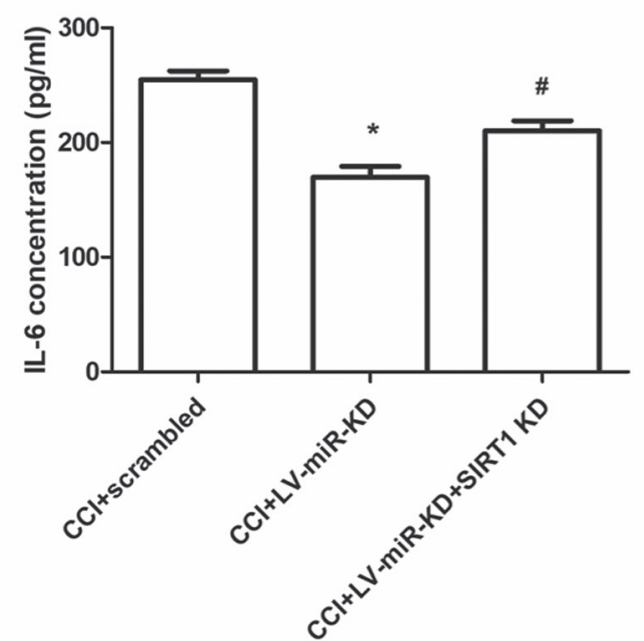

B

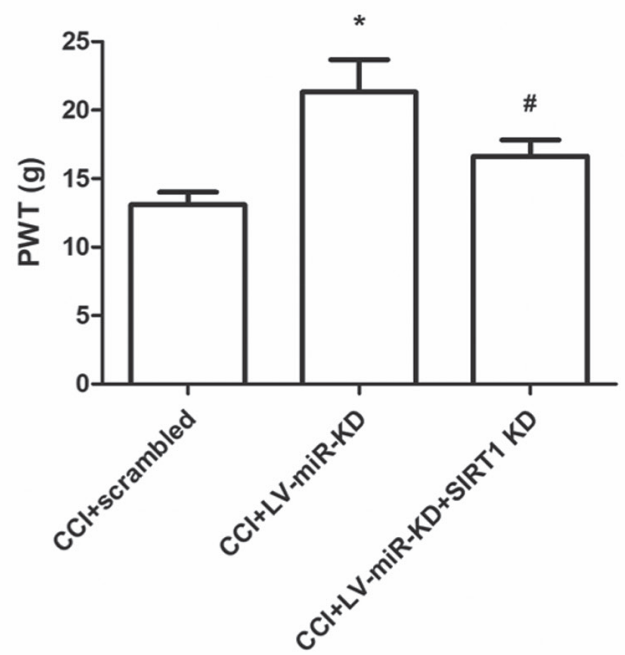

D

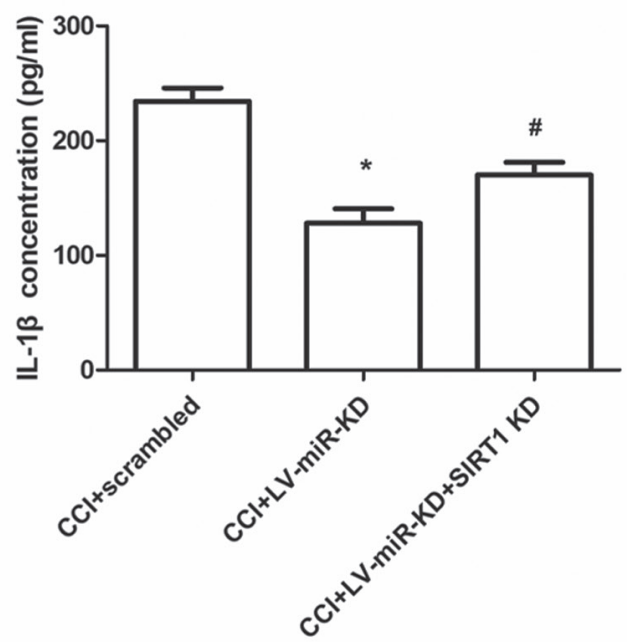

$\mathbf{F}$

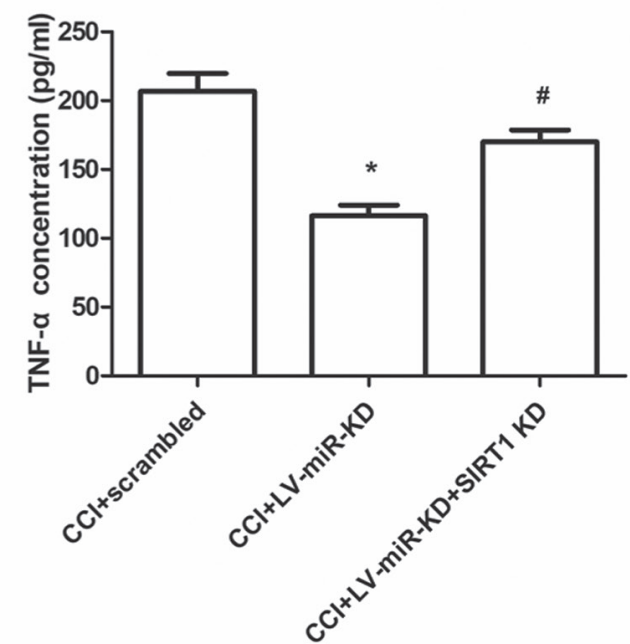

Figure 5. SIRT1 abrogated the effect of miR-448 on neuropathic pain development. miR-448 inhibitor lentivirus, or miR-448 inhibitor and shSIRT1 lentivirus, were co-infected into CCI rats. (A) SIRT1 mRNA expression in CCI rats. (B) Mechanical allodynia as assessed by the PWT. (C) Thermal hyperalgesia as assessed by the PWL. The protein levels of (D) IL-1 $\beta,(E)$ IL- 6 and (F) TNF- $\alpha$ in the L4-L6 spinal cords of rats were measured by ELISA on postoperative day 7. ${ }^{*} \mathrm{P}<0.05$ vs. the CCI + scrambled group; ${ }^{\#} \mathrm{P}<0.05$ vs. the CCI + LV-miR-KD group. SIRT1, sirtuin 1; miR, microRNA, sh, short hairpin; CCI, chronic constriction injury; PWT, paw withdrawal threshold; PWL, paw withdrawal latency; IL, interleukin; TNF- $\alpha$, tumor necrosis factor- $\alpha$; LV, lentivirus; KD, knockdown.

In conclusion, the present study demonstrated that miR-448 promoted the development of neuropathic pain in a rat model of CCI by regulating neuroinflammation via SIRT1. Therefore, miR-448 may serve a novel target for neuropathic pain treatment. 


\section{Acknowledgements}

Not applicable.

\section{Funding}

No funding was received.

\section{Availability of data and materials}

The datasets used and/or analyzed during the current study are available from the corresponding author on reasonable request.

\section{Authors' contributions}

$\mathrm{XW}$ and YC designed the study, and contributed to the writing of the manuscript. YC and WG performed all the experiments, analyzed the data, and contributed significantly to the revision of the manuscript. All authors read and approved the final manuscript.

\section{Ethics approval and consent to participate}

The experimental procedures for care and use of animals were approved by the Medical Ethics Committee of Shengli Oilfield Central Hospital.

\section{Patient consent for publication}

Not applicable.

\section{Competing interests}

The authors declare that they have no competing interests.

\section{References}

1. Zhao Y and Yang Z: Effect of Wnt signaling pathway on pathogenesis and intervention of neuropathic pain. Exp Ther Med 16 3082-3088, 2018.

2. Sommer C, Leinders $M$ and Üçeyler $N$ : Inflammation in the pathophysiology of neuropathic pain. Pain 159: 595-602, 2018.

3. Bouhassira D and Attal N: Emerging therapies for neuropathic pain: New molecules or new indications for old treatments? Pain 159: 576-582, 2018.

4. Lema MJ, Foley KM and Hausheer FH: Types and epidemiology of cancer-related neuropathic pain: The intersection of cancer pain and neuropathic pain. Oncologist 15 (Suppl 2): S3-S8, 2010.

5. Jiang YR, Du JY, Wang DD and Yang X: miRNA-130a improves cardiac function by down-regulating TNF- $\alpha$ expression in a rat model of heart failure. Eur Rev Med Pharmacol Sci 22 8454-8461, 2018.

6. Mao Q, Chen C, Liang H, Zhong S, Cheng X and Li L: Astragaloside IV inhibits excessive mesangial cell proliferation and renal fibrosis caused by diabetic nephropathy via modulation of the TGF- $\beta 1 / \mathrm{Smad} / \mathrm{miR}-192$ signaling pathway. Exp Ther Med 18: 3053-3061, 2019

7. Meng Y, Shang F and Zhu Y: miR-124 participates in the proliferation and differentiation of brain glioma stem cells through regulating Nogo/NgR expression. Exp Ther Med 18: 2783-2788, 2019.

8. Wang JR, Liu B, Zhou L and Huang YX: MicroRNA-124-3p suppresses cell migration and invasion by targeting ITGA 3 signaling in bladder cancer. Cancer Biomark 24: 159-172, 2019.

9. Wang J, Chu Y, Xu M, Zhang X, Zhou Y and Xu M: miR-21 promotes cell migration and invasion of hepatocellular carcinoma by targeting KLF5. Oncol Lett 17: 2221-2227, 2019.
10. Qiu T, Wang K, Li X and Jin J: miR-671-5p inhibits gastric cancer cell proliferation and promotes cell apoptosis by targeting URGCP. Exp Ther Med 16: 4753-4758, 2018.

11. Zhang Y, Su Z, An LJ, Li L, Wei M, Ge DJ and Liu HL: miR-98 acts as an inhibitor in chronic constriction injury-induced neuropathic pain via downregulation of high-mobility group AT-hook 2. J Cell Biochem 120: 10363-10369, 2019.

12. Wen J, He T, Qi F and Chen H: MiR-206-3p alleviates chronic constriction injury-induced neuropathic pain through targeting HDAC4. Exp Anim 68: 213-220, 2019.

13. Bao Y, Wang S, Xie Y, Jin K, Bai Y and Shan S: MiR-28-5p relieves neuropathic pain by targeting Zeb1 in CCI rat models. J Cell Biochem 119: 8555-8563, 2018.

14. Lu Y, Jian MY, Ouyang YB and Han RQ: Changes in rat brain MicroRNA expression profiles following sevoflurane and propofol anesthesia. Chin Med J (Engl) 128: 1510-1515, 2015.

15. Bennett GJ and Xie YK: A peripheral mononeuropathy in rat that produces disorders of pain sensation like those seen in man. Pain 33: 87-107, 1988.

16. Schmalbruch H: Fiber composition of the rat sciatic nerve. Anat Rec 215: 71-81, 1986.

17. Livak KJ and Schmittgen TD: Analysis of relative gene expression data using real-time quantitative PCR and the 2(-Delta Delta C(T)) method. Methods 25: 402-408, 2001.

18. Ni HD, Xu LS, Wang Y, Li H, An K, Liu M, Liu Q, Deng H, He Q, Huang B, et al: Astrocyte activation in the periaqueductal gray promotes descending facilitation to cancer-induced bone pain through the JNK MAPK signaling pathway. Mol Pain 15: $1744806919831909,2019$.

19. Degu H, Wondimagegnehu A, Yifru YM and Belachew A: Is health related quality of life influenced by diabetic neuropathic pain among type II diabetes mellitus patients in Ethiopia? PLoS One 14: e0211449, 2019.

20. Kanda H, Liu S, Kanao M, Yi H, Iida T, Huang W, Kunisawa T, Lubarsky DA and Hao S: Gene therapy with HSV encoding p55TNFR gene for HIV neuropathic pain: An evidence-based mini-review. Transl Perioper Pain Med 2: 24-32, 2017.

21. Tilley DM, Cedeño DL, Kelley CA, DeMaegd M, Benyamin R and Vallejo R: Changes in dorsal root ganglion gene expression in response to spinal cord stimulation. Reg Anesth Pain Med 42: 246-251, 2017.

22. Safieh-Garabedian B, Nomikos M and Saadé N: Targeting inflammatory components in neuropathic pain: The analgesic effect of thymulin related peptide. Neurosci Lett 702: 61-65, 2019.

23. Chen P, Li Y, Li L, Yu Q, Chao K, Zhou G, Qiu Y, Feng R, Huang S, He Y, et al: Circulating microRNA146b-5p is superior to C-reactive protein as a novel biomarker for monitoring inflammatory bowel disease. Aliment Pharmacol Ther 49: 733-743, 2019.

24. Jia QW, Chen ZH, Ding XQ, Liu JY, Ge PC, An FH, Li LH, Wang LS, Ma WZ, Yang ZJ and Jia EZ: Predictive effects of circulating miR-221, miR-130a and miR-155 for coronary heart disease: A multi-ethnic study in China. Cell Physiol Biochem 42: 808-823, 2017.

25. Su HY, Lin ZY, Peng WC, Guan F, Zhu GT, Mao BB, Dai B, Huang $\mathrm{H}$ and Hu ZQ: MiR-448 downregulates CTTN to inhibit cell proliferation and promote apoptosis in glioma. Eur Rev Med Pharmacol Sci 22: 3847-3854, 2018.

26. Zhang R, Sui L, Hong X, Yang M and Li W: MiR-448 promotes vascular smooth muscle cell proliferation and migration in through directly targeting MEF2C. Environ Sci Pollut Res Int 24: 22294-22300, 2017.

27. Hong X, Xu Y, Qiu X, Zhu Y, Feng X, Ding Z, Zhang S, Zhong L, Zhuang Y, Su C, et al: MiR-448 promotes glycolytic metabolism of gastric cancer by downregulating KDM2B. Oncotarget 7: 22092-22102, 2016.

28. Wang Y, Pang QJ, Liu JT, Wu HH and Tao DY: Down-regulated miR-448 relieves spinal cord ischemia/reperfusion injury by up-regulating SIRT1. Braz J Med Biol Res 51: e7319, 2018.

29. An J, Cai T, Che H, Yu T, Cao Z, Liu X, Zhao F, Jing J, Shen X, Liu M, et al: The changes of miRNA expression in rat hippocampus following chronic lead exposure. Toxicol Lett 229: 158-166, 2014.

30. Myers RR, Campana WM and Shubayev VI: The role of neuroinflammation in neuropathic pain: Mechanisms and therapeutic targets. Drug Discov Today 11: 8-20, 2006.

31. Fei H, Shi M, Chen L, Wang Z and Suo L: MicroRNA-18 promotes apoptosis of islet $\beta$-cells via targeting NAV1. Exp Ther Med 18: 389-396, 2019.

32. Li QY, Xu HY and Yang HJ: Effect of proinflammatory factors TNF- $\alpha$, IL-1 $\beta$, IL- 6 on neuropathic pain. Zhongguo Zhong Yao Za Zhi 42: 3709-3712, 2017 (In Chinese). 
33. Li HL, Sun JJ, Ma H, Liu SJ, Li N, Guo SJ, Shi Y, Xu YY, Qi ZY, Wang YQ, et al: MicroRNA-23a inhibits endometrial cancer cell development by targeting SIX1. Oncol Lett 18: 3792-3802, 2019.

34. Wang S, Li C, Yu Y and Qiao J: Decreased expression of microRNA-145 promotes the biological functions of fibroblasts in hypertrophic scar tissues by upregulating the expression of transcription factor SOX-9. Exp Ther Med 18: 3450-3460, 2019.

35. Yang $\mathrm{H}, \mathrm{Wu} \mathrm{D}, \mathrm{Li} \mathrm{H}$, Chen $\mathrm{N}$ and Shang Y: Downregulation of microRNA-448 inhibits IL-1 $\beta$-induced cartilage degradation in human chondrocytes via upregulation of matrilin-3. Cell Mol Biol Lett 23: 7, 2018.

36. Li B, Ge L, Li M, Wang L and Li Z: miR-448 suppresses proliferation and invasion by regulating IGF1R in colorectal cancer cells. Am J Transl Res 8: 3013-3022, 2016.

37. Fujita Y and Yamashita T: Sirtuins in neuroendocrine regulation and neurological diseases. Front Neurosci 12: 778, 2018.

38. Zhao H, Chen S, Gao K, Zhou Z, Wang C, Shen Z, Guo Y, Li Z, Wan Z, Liu C and Mei X: Resveratrol protects against spinal cord injury by activating autophagy and inhibiting apoptosis mediated by the SIRT1/AMPK signaling pathway. Neuroscience 348 : 241-251, 2017.
39. Zhang Z, Ding X, Zhou Z, Qiu Z, Shi N, Zhou S, Du L, Zhu X, Wu Y, Yin X and Zhou C: SIRT1 alleviates diabetic neuropathic pain by regulating synaptic plasticity of spinal dorsal horn neurons. Pain 160: 1082-1092, 2019.

40. Cui C, Li Y and Liu Y: Down-regulation of miR-377 suppresses high glucose and hypoxia-induced angiogenesis and inflammation in human retinal endothelial cells by direct up-regulation of target gene SIRT1. Hum Cell 32: 260-274, 2019.

41. Chang YC, Lin CW, Hsieh MC, Wu HJ, Wu WS, Wu WC and Kao YH: High mobility group B1 up-regulates angiogenic and fibrogenic factors in human retinal pigment epithelial ARPE-19 cells. Cell Signal 40: 248-257, 2017.

(c) (i) () $€$ This work is licensed under a Creative Commons International (CC BY-NC-ND 4.0) License. 